\title{
Femtosecond wave-packet revivals in ozone
}

T. Latka, ${ }^{1,2, *}$ V. Shirvanyan, ${ }^{1,2}$ M. Ossiander, ${ }^{1,2}$ O. Razskazovskaya, ${ }^{2,3}$ A. Guggenmos, ${ }^{2,3}$ M. Jobst,,${ }^{1,2}$ M. Fieß, $, 2,3$ S. Holzner, ${ }^{2,3}$ A. Sommer, ${ }^{1,2,3}$ M. Schultze, ${ }^{2,3}$ C. Jakubeit,,${ }^{2,3}$ J. Riemensberger, ${ }^{1}$ B. Bernhardt, ${ }^{1}$ W. Helml, ${ }^{1,3}$ F. Gatti, ${ }^{4,5}$ B. Lasorne, ${ }^{4}$ D. Lauvergnat, ${ }^{6}$ P. Decleva, ${ }^{7}$ G. J. Halász, ${ }^{8}$ Á. Vibók,,${ }^{9,10}$ and R. Kienberger ${ }^{1,2,{ }^{\prime}}$

${ }^{1}$ Physik-Department, Technische Universität München, James-Franck-Straße 1, 85748 Garching, Germany

${ }^{2}$ Max-Planck-Institut für Quantenoptik, Hans-Kopfermann-Straße 1, 85748 Garching, Germany

${ }^{3}$ Fakultät für Physik, Ludwig-Maximilians-Universität München, Am Coulombwall 1, 85748 Garching, Germany

${ }^{4}$ Institut Charles Gerhardt, CNRS, Université de Montpellier, F-34095 Montpellier, France

${ }^{5}$ Institut des Sciences Moléculaires d'Orsay (UMR 8214), CNRS, Université Paris-Sud/Paris-Saclay, F-91405 Orsay, France

${ }^{6}$ Laboratoire de Chimie Physique, CNRS, Université Paris-Sud, F-91405 Orsay, France

${ }^{7}$ Dipartimento di Scienze Chimiche e Farmaceutiche, Università di Trieste, Via L. Giorgieri 1, 34127 Trieste, Italy

${ }^{8}$ Department of Information Technology, University of Debrecen, H-4002 Debrecen, PO Box 400, Hungary

${ }^{9}$ Department of Theoretical Physics, University of Debrecen, H-4002 Debrecen, PO Box 400, Hungary

${ }^{10}$ ELI-ALPS, ELI-HU Non-Profit Ltd, Dugonics tér 13, H-6720 Szeged, Hungary

(Received 12 October 2018; published 10 June 2019)

\begin{abstract}
Photodissociation of ozone following absorption of biologically harmful solar ultraviolet radiation is the key mechanism for the life protecting properties of the atmospheric ozone layer. Even though ozone photolysis is described successfully by post-Hartree-Fock theory, it has evaded direct experimental access so far, due to the unavailability of intense ultrashort deep ultraviolet radiation sources. The rapidity of ozone photolysis with predicted values of a few tens of femtoseconds renders both ultrashort pump and probe pulses indispensable to capture this manifestation of ultrafast chemistry. Here, we present the observation of femtosecond time-scale electronic and nuclear dynamics of ozone triggered by $\sim 10-\mathrm{fs}, \sim 2-\mu \mathrm{J}$ deep ultraviolet pulses and, in contrast to conventional attochemistry experiments, probed by extreme ultraviolet isolated pulses. An electronic wave packet is first created. We follow the splitting of the excited $B$-state related nuclear wave packet into a path leading to molecular fragmentation and an oscillating path, revolving around the Franck-Condon point with 22-fs wave-packet revival time. Full quantum-mechanical ab initio multiconfigurational time-dependent Hartree simulations support this interpretation.
\end{abstract}

DOI: 10.1103/PhysRevA.99.063405

\section{INTRODUCTION}

Ozone is one of the best studied triatomic molecules [1-6] as it is essential for life on Earth. Its importance to the global community stems from the property of the atmospheric ozone layer to efficiently absorb genetically harmful deep ultraviolet (DUV) solar radiation. Efforts regarding its preservation culminated in the internationally ratified Montreal Protocol that aims at reducing the amount of potentially ozone-depleting substances, still not covering all of these, but which is perpetually amended by the inclusion of additional chemical compounds whose negative impact on the ozone layer is revealed [7].

Besides the influence of these substances on ozone depletion, ozone photodissociation is the most direct mechanism and the one responsible for the protective function of the atmospheric ozone layer. It occurs following the one-photon transition between the ground $X\left(1^{1} A_{1}\right)$ electronic state and the excited $B\left(1^{1} B_{2}\right)$ electronic state within the DUV spectral

\footnotetext{
*tobias.latka@mytum.de

†reinhard.kienberger@tum.de
}

domain of the strongly absorbing Hartley band (200-300 nm or 6.2-4.1 eV) according to $\mathrm{O}_{3}+h v \rightarrow \mathrm{O}_{3}{ }^{*} \rightarrow \mathrm{O}_{2}+\mathrm{O}$.

In 2013, Halász [8] et al. predicted theoretically that the formation of a coherent electronic nuclear wave packet (NWP) consisting of $X$ and $B$ states is created when ozone is irradiated with an ultrashort DUV pulse. The electronic (neutral) wave packet is a superposition of the $X$ and $B$ states leading to an oscillation of the electronic charge density from one $\mathrm{O}-\mathrm{O}$ bond to the other on the subfemtosecond time scale (with a period of $0.9 \mathrm{fs}$ ). This wave packet is an alternating superposition of two resonant forms, $(\mathrm{O}-\mathrm{O}=\mathrm{O} \leftrightarrow \mathrm{O}=$ $\mathrm{O}-\mathrm{O}$ ), each having an excess or lack of electrons on one or the other bond. These chemical structures are precursors of the two equivalent dissociation channels to $\mathrm{O}+\mathrm{O}_{2}$ or $\mathrm{O}_{2}+\mathrm{O}$ (see Fig. 1). The nuclear oscillations in the $B$ state result in a coherence revival between the electronic states before molecular fragmentation is completed (see Fig. 1 for times greater than $20.1 \mathrm{fs}$ and Sec. 13 of the Supplemental Material [9] for further information on the underlying calculation).

Throughout this process, the electronic coherence between the $X$ and $B$ states is thus not destroyed as one would intuitively expect due to dephasing between components of NWPs on different potential-energy surfaces (PESs) upon nuclear motion $[10,11]$. The wave-packet recurrence can be 
(a)

(b)

$-10.1 \mathrm{fs}$

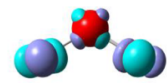

$-9.7 \mathrm{fs}$
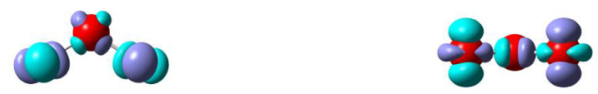

-9.2 fs
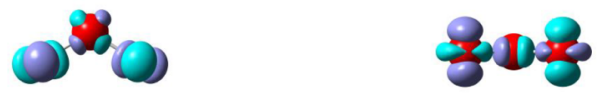

$0.1 \mathrm{fs}$

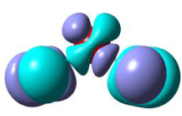

$0.5 \mathrm{fs}$

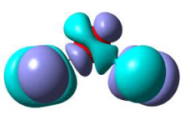

$1.0 \mathrm{fs}$

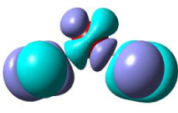

$20.1 \mathrm{fs}$

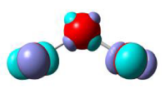

20.6 fs

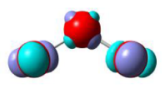

$21.0 \mathrm{fs}$
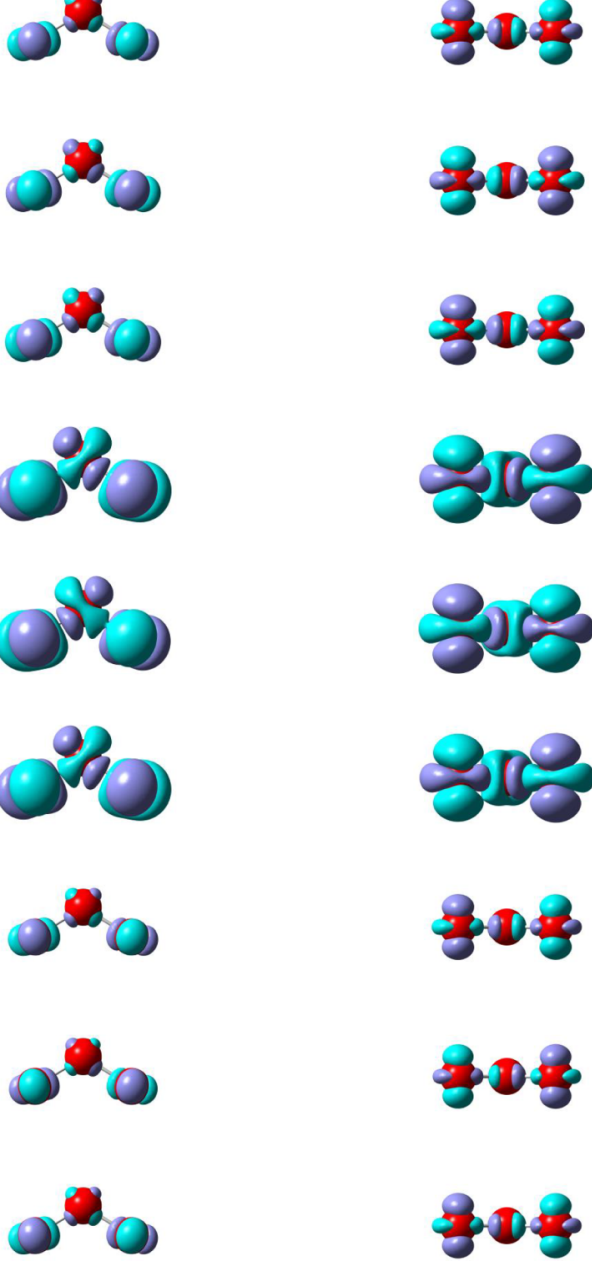

FIG. 1. (a) Top view and (b) side view of the differential timedependent one-electron reduced density between the excited $B$ state and the ground $X$ state of ozone at the Franck-Condon point. After interaction of the ozone molecule with a $\sim 10$-fs, $\sim 2-\mu \mathrm{J}$ DUV pulse, as used in the present work, the electron cloud starts oscillating between the two bonds with a periodicity of only $0.9 \mathrm{fs}$. This is equivalent to an alternating excess or lack of electrons on either side of the ozone molecule and is considered a precursor of the ozone photodissociation. Oxygen atoms of the ozone molecule (red spheres). Lack of electronic density (light blue surface isovalue: -0.0001 a.u.); excess of electronic density (light purple surface isovalue: +0.0001 a.u.). The moment of maximum $B$-state excitation coincides with $0 \mathrm{fs}$. Electronic charge densities for times greater than 20.1 fs originate from a coherence revival associated with the $B$-state PES dynamics, as discussed in the main text.

interpreted as a part of the $B$-state wave function returning to the Franck-Condon (FC) point along its mean trajectory. Although the direct experimental verification of this coherence revival is not straightforward (and impossible with the available means), its occurrence can indirectly be deduced from a characteristic feature appearing in the temporal evolution of the energy-integrated number of $B$-state-related photoelectrons in time-resolved photoelectron spectroscopy [12]. In contrast to the majority of pump-probe experiments regarding the investigation of ultrafast dynamical processes in molecules, the photoelectrons created in this experiment originate from photoionization of valence electrons by extreme ultraviolet (XUV) isolated pulses rather than by high-field infrared femtosecond pulses. Consequently, our probing step deviates from the original approach frequently pursued in attochemistry experiments. Here, we do not utilize attosecond XUV pulses for producing coherent superpositions of multiple electronic states, but for probing. The current approach is thus advantageous over the usual procedure, as probing with the XUV pulse negligibly distorts the molecular dynamics due to a much smaller ponderomotive energy, allowing for their field-free detection [13].

The coherent wave packet dynamics of $\mathrm{O}_{3}$ and its strong DUV Hartley band (one of the most intense among all molecules) make it an ideal candidate for studying these effects experimentally. Despite the numerous advantages of ozone, we want to state that its synthesis and temporary storage enforce special precautions as ozone is prone to environmental disturbances like stray light absorption, vibrations and-as outlined above-reactions with specific chemical compounds, all of them triggering its sudden conversion into oxygen with considerable detonation velocity rivalling that of modern explosives.

In this article, we report on both the creation of a coherent electronic NWP in neutrally charged ozone and the first realtime observation of its subsequent femtosecond time-scale photochemistry. The latter comprise rapid nuclear vibrations in the excited $B$ state as well as ultrafast dissociation, similar to that recently observed in gaseous $\mathrm{HCl}$ via core-hole creation, albeit here in a neutral polyatomic molecule (see Fig. 2) [14]. The associated vibration period is determined via time-resolved photoelectron spectroscopy experiments to be $(22.6 \pm 1.7)$ fs and is compared to theory, where the uncertainty refers to the $95 \%$ confidence interval. It is noteworthy that, until today, the theoretical description and computational treatment of the coupled nuclear and electronic dynamics of such a medium-sized molecular system like $\mathrm{O}_{3}$ is still a very demanding task. However, our setup pushes the frontier of ultrafast pump-probe experiments towards the DUV spectral domain, paving the way for few-femtosecond or even attosecond investigations of other DUV absorbing molecules. Among those, biomolecules such as peptides [15], proteins [16], or DNA [17] are of utmost interest owing to their biological significance.

\section{MEASUREMENT OF ELECTRONIC AND NUCLEAR DYNAMICS}

In time-resolved photoelectron spectroscopy experiments, investigation of the electronic and nuclear dynamics [13] of ozone relies on the measurement of chemical shifts that are imprinted on the kinetic energy of valence electrons, released by a high-photon-energy XUV probe pulse. These dynamics are a manifestation of certain electronic states being either populated or depleted under the impact of a resonant DUV pump pulse. Besides the electronic contribution to the photoelectrons' kinetic-energy modifications, they can also be attributed to the induced electronic and nuclear dynamics and 


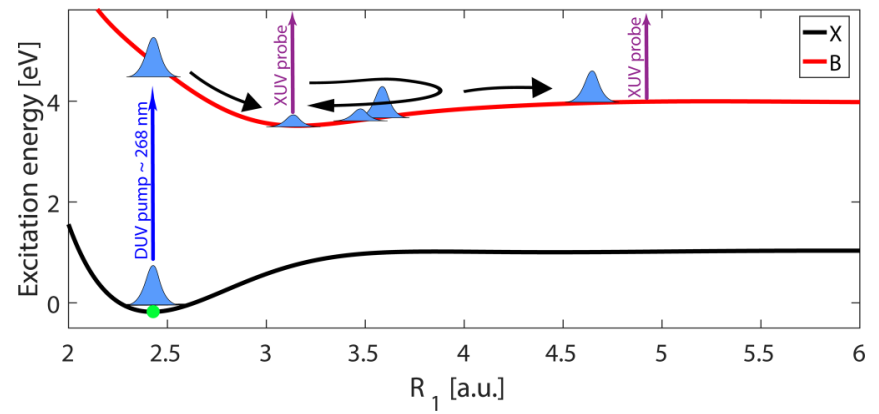

FIG. 2. Sketch of the $X$ state (black solid line) and $B$ state (red solid line) related PESs of ozone as a function of the dissociative degree of freedom, $R_{1}$. The vibrational, $R_{2}$, and bending, $\alpha$, degrees of freedom were kept constant $\left(R_{2}=2.42\right.$ a.u.; $\left.\alpha=116.9^{\circ}\right)$. The NWP in the ground state (bottommost Gaussian) is promoted from the ground $X$ state into the excited $B$ state (vertical blue arrow; uppermost Gaussian) at the FC point (green dot) and starts moving down the slope of the $B$-state PES, continuously probed by an XUV probe pulse (vertical purple arrows indicate photoionization at different delay times during the evolution of the molecular dynamics). Midway between the FC point (equilibrium position at $R_{1}=2.42$ a.u.) and the outer range of the PES the NWP bifurcates where part of it returns to the FC point while the other part dissociates (rightmost Gaussian). It should be noted that the superposition of the two electronic $X$ and $B$ states can be considered as an oscillating electronic wave packet, since the $B$-state component contributes a very fast oscillating phase with regard to the $X$ state component (see Fig. 1).

vary with time as the launched NWP moves according to the topography of the corresponding electronic PES.

A cut through those PESs along the reaction coordinatethe dissociative degree of freedom, $R_{1}$, connecting the central $\mathrm{O}$ atom with either one of the terminal $\mathrm{O}$ atoms - together with the underlying dynamics is depicted in Fig. 2 for the two experimentally relevant states of ozone. The black solid line marks the ground $X\left(1{ }^{1} A_{1}\right)$ electronic state of ozone where the molecules reside before being exposed to the pump (bottommost Gaussian). The red solid line, by contrast, visualizes the excited $B\left({ }^{1} B_{2}\right)$ electronic state that is populated (vertical blue arrow) once the pump interacts with the molecule. In Fig. 2 the two remaining degrees of freedom - the vibrational coordinate $R_{2}$ and the opening angle $\alpha$ between the central and the two outermost oxygen atomswere fixed $\left(R_{2}=2.42\right.$ a.u.; $\left.\alpha=116.9^{\circ}\right)$ and correlate with the ground-state equilibrium configuration of ozone (the green dot in Fig. 2 depicts the FC point). The distinct energy separation between these PESs $(\sim 4.6 \mathrm{eV}$, corresponding to a $\sim 268-\mathrm{nm}$ excitation wavelength) facilitates the experimental determination of population transfer between these states whose temporal evolution, in turn, can be related to the intramolecular dynamics. Hence, energy-related superpositions of photoelectron wave packets emanating from different states are hardly expected, and the respective features can be assigned unambiguously to their respective state.

Despite the sufficiently large $X \rightarrow B$ transition energy, the bandwidth of the ionizing XUV probe pulse must not exceed the latter to ensure the discriminability between the groundand excited-state-related photoelectrons in the time-resolved photoelectron spectroscopy experiment, and in turn limits the achievable temporal resolution. Therefore, preliminary calculations based upon a Gaussian convolved stick spectrum of ozone cation states taken from Ref. [18] were performed, neglecting the rovibrational structure of the spectrum of each photoionization channel. Our simulations suggest that an energy resolution of $1.1 \mathrm{eV}$ is adequate to discern between both states, which is equivalent to an XUV full width at half maximum (FWHM) pulse duration Fourier limit of $\sim 1.7$ fs centered around $99.5 \mathrm{eV}$. Experimentally we generate the XUV prior to the DUV via high harmonic generation (HHG) in a quasistatic gas cell filled with neon and exposed to a carrierenvelope phase-stabilized near-infrared (NIR) sub-4-fs laser pulse (see Secs. 1 and 7 of the Supplemental Material [9]).

Figure 3(a) illustrates the generation setup of intense $\sim 10$ fs DUV pulses [19-22] along one arm of a Mach-Zehnder interferometer for pump-probe spectroscopy and which is based upon type-I second harmonic generation (SHG) of spectrally broadened NIR pulses (see Secs. 2-5 of the Supplemental Material [9] for a more detailed description of the DUV generation, including a temporal characterization of the DUV). Figure 3(b) depicts the ozone experiment itself that makes use of a crossed-polarization collinear XUV-DUV beam geometry. The gaseous ozone sample comes from a reservoir of synthesized and highly pure ( $\sim 99 \%)$ ozone (see Sec. 6 of the Supplemental Material [9]).

In our experiment, we forgo molecular alignment, since the molecular $y$ component is the only nonvanishing element of the $X \rightarrow B$ transition dipole moment. This implies that the molecule's excitation is restricted to polarizations that possess a component along this direction [red molecule in Fig. 3(b)] whereas otherwise it remains largely unaffected [blue molecule in Fig. 3(b)] rendering molecular alignment expendable.

Additionally, Decleva [18] et al. recently investigated the photodynamics of ozone and predicted its signature in terms of time-resolved photoelectron spectroscopy experiments. Concerning the differential photoionization cross section of randomly oriented ozone for 95-eV XUV probe photons, they report an asymmetry parameter of $\beta \approx 2$ for all ionizations [23]. This asymmetry is a sequitur of photoionization selection rules [24] governing the observability of excited state-related photoelectrons preceding dissociation and partly motivates the crossed XUV-DUV polarizations in our experiment (see Sec. 8 of the Supplemental Material [9] for group-theoretical aspects).

Detection of the final photoelectron momentum along the XUV polarization axis, recorded for different relative delay times $\tau$ between DUV and XUV, yields a spectrogram including contributions of intramolecular dynamics in ozone. The estimated effective peak intensity of the DUV amounts to $(1.8 \pm 1.0) \times 10^{12} \mathrm{~W} / \mathrm{cm}^{2}$ on target resulting in a $40 \%$ excitation probability from $X$ to $B$ for aligned molecules (the $B$-state excitation profile for a 10 -fs DUV pulse is shown in Sec. 10 of the Supplemental Material [9]).

\section{DIRECT OBSERVATION OF OZONE PHOTODISSOCIATION}

The combined spectrogram is obtained by time-ordered concatenation of all measured photoelectron spectra as shown 
(a)

(b)

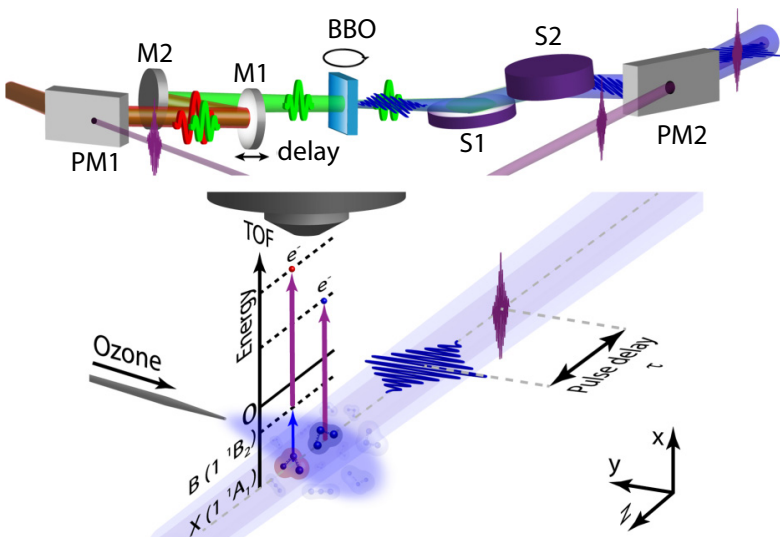

FIG. 3. (a) Sketch of the DUV generating type-I SHG setup as used in the experiment along with the pump-probe delay line in one arm of the Mach-Zehnder interferometer: The spectrally broadened NIR pulse is separated from the XUV at the first perforated mirror (PM1). Subsequently, it is focused via a protected Ag mirror (M1) into a rotatable, free-standing $20-\mu \mathrm{m}$-thin $\mathrm{BBO}$ crystal $\left(\theta=44.3^{\circ}\right.$, $\varphi=90^{\circ}$ ) and NIR attenuated via a broadband, spectrally selective dielectric mirror (M2) before the DUV and XUV get recombined in a collinear fashion via the second perforated mirror (PM2) at the exit of the interferometer. The silicon wafer chicane (S1/S2) put at the NIR Brewster angle $\left(75^{\circ}\right.$ angle of incidence) ensures both NIR reduction and high DUV throughput due to crossed polarizations of DUV and NIR, the latter being attenuated by more than four orders of magnitude. Another DUV reflecting, but NIR attenuating dielectric mirror in front of the second perforated mirror (PM2) is not shown. (b) Setup of the time-resolved photoelectron spectroscopy experiment for measuring electronic and nuclear dynamics in ozone: A highly pure ozone sample enters the interaction region below the electron time-of-flight detector (TOF) through a graphite coated glass nozzle perpendicular to the TOF axis orientation and the cross-polarized, collinear DUV-XUV-pulse train (along the $y$ axis). The DUV pulse is used to excite the ozone molecules from the ground $X$ state into the excited $B$ state, whereas the XUV continuously ionizes the ozone molecules at different delay times to keep track of the evolving molecular dynamics. Electronic excitation from $X \rightarrow B$ will only be accomplished if the DUV polarization points in the same direction as the molecule's dipole moment along the $y$ axis (red molecule) and will be thoroughly suppressed in all other cases of molecular orientation (blue molecule). The XUV released photoelectrons and their kinetic energy are measured with the TOF as a function of pulse delay $\tau$ between DUV and XUV. Here, $\tau>0$ refers to the situation where the DUV pump precedes the XUV probe pulse and vice versa.

in Fig. 4(a) using a logarithmic color scale. Negative time delays mean the XUV precedes the DUV and vice versa. Zero delay is set to coincide with maximum $B$-state population and kept fixed throughout all following plots. On the left-hand side, we show a typical $\mathrm{O}_{3}$ photoelectron spectrum taken with the XUV only (ionizing from the $X$ state, without pumping the $B$ state), as well as the four most important energy domains used for data evaluation (gray horizontal bars). Refer to Fig. S8 (Sec. 7 of the Supplemental Material [9]) for the experimental spectra of $\mathrm{O}_{3}$ and $\mathrm{O}_{2}$. The uppermost bar $(90-93 \mathrm{eV})$ in Fig. 4(a) belongs to cation states corresponding to the $B$ state. The second (86.5-88 eV) and fourth (79.5-81 eV) energy domains (counted from the top) both refer to ground-state- associated cation states (originating from energy-bunched ground-state-related cation states), whereas the third energy range $(82.5-85 \mathrm{eV})$ covers photoelectrons expected to emanate from pure diatomic oxygen $\mathrm{O}_{2}$ (an ozone spectrum overlaid with a calculated stick spectrum of cation states can be found in Sec. 7 of the Supplemental Material [9]).

To obtain the state-specific temporal evolution of the number of photoelectrons [Figs. 4(b), 4(c), and 5(a)] we pursue a multilevel data analysis scheme whose details are given in Sec. 12 of the Supplemental Material [9]. In the process, a function consisting of a Gaussian convolved superposition of a cosine and an exponentially decaying function are fitted to the data. The latter two functions account for the dissociative and oscillating nature of the excited-state wave function as will soon become apparent. In all subsequent figures, vertical error bars and red shadings refer to the $95 \%$ confidence interval for the data points and the fit, respectively, whereas horizontal error bars indicate the estimated maximum experimental uncertainty in the temporal assignment of a data point.

Finally, Figs. 4(b) and 4(c) summarize the temporal evolution of the energy-integrated number of photoelectrons originating from the ground state [Fig. 4(b)] and diatomic oxygen $\mathrm{O}_{2}$ [Fig. 4(c)].

Figure 4(b) shows clear evidence for a gradual depletion of ground-state molecules undergoing a DUV-induced transition to the weakly binding dissociative $B$ state. The decay starts at $-10 \mathrm{fs}$ and lasts until $10 \mathrm{fs}$, followed by an intermediate increase of ground-state molecules around $25 \mathrm{fs}$, before their number starts dropping again till the end of the sampled time interval. Accompanying the groundstate depletion is the $\sim 20$-fs delayed rise in the number of photoelectrons originating from the $\mathrm{O}_{2}$ energy domain $(82.5-85 \mathrm{eV})$ observed in Fig. 4(c). We attribute this to the formation of diatomic oxygen, $\mathrm{O}_{2}$, confirmed by comparison to a photoelectron spectrum recorded in oxygen in the absence of ozone. Parts of its most prominent feature fall between those of the ozone photoelectron spectrum and can thus be identified unambiguously with the dissociation of ozone into diatomic oxygen. Nevertheless, the peak contribution of the $\mathrm{O}_{2}$ spectrum detrimentally overlaps with the most intense part of the ground-state-related $\mathrm{O}_{3}$ spectrum and overcompensates its decrease. This partly explains the monitored temporary rise around 25 fs in Fig. 4(b) (see Sec. 11 of the Supplemental Material [9]). However, our measurement reveals the molecular fragmentation to be completed after only $20 \mathrm{fs}$, marking a clear manifestation of ultrafast chemistry.

\section{NUCLEAR AND ELECTRONIC DYNAMICS IN OZONE}

Having found ozone photodissociation to happen on such a rapid time scale, we now focus on the excited $B$ state's inherent temporal evolution expecting a comparable time scale, which is shown in Fig. 5(a). Obviously, the profile does exhibit additional structure compared to a Gaussian convolved decay function. The excitation builds up within $\sim 20 \mathrm{fs}$, forcing the electron cloud to oscillate as the DUV generates a coherent superposition of $X$ and $B$ states (see Fig. 1). Fitting the data using the routine detailed in the Supplemental Material [9] yields a FWHM DUV duration within the 95\% confidence interval of $\tau_{\mathrm{DUV}}=(12.4 \pm 2.3)$ fs and a decay time constant for the 


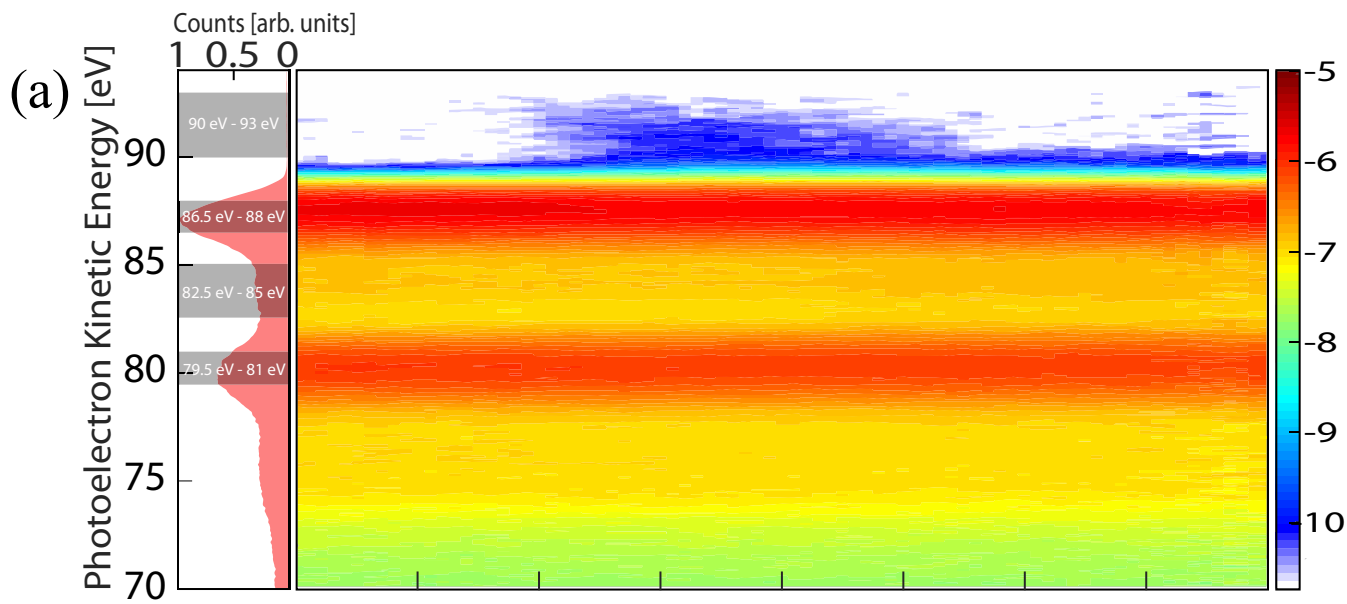

(b)

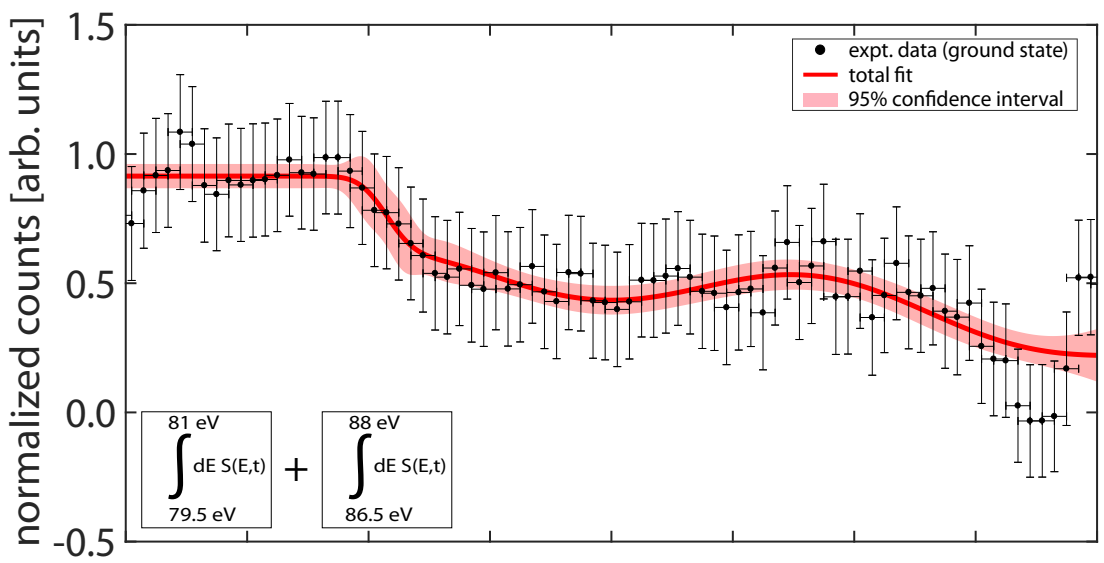

(c)

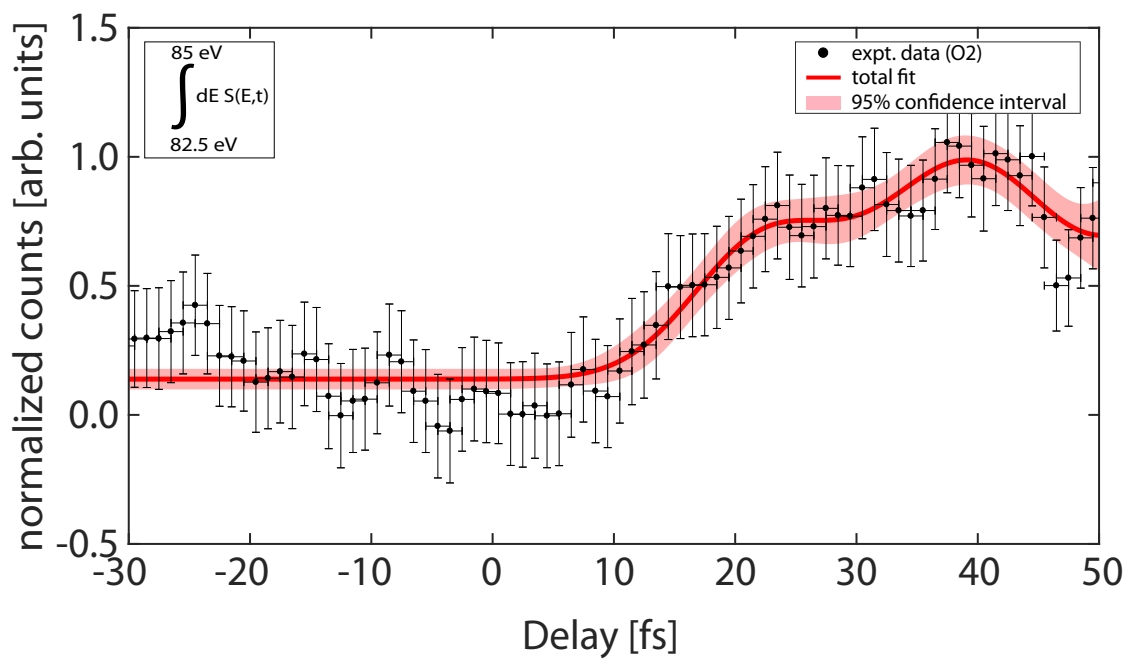

FIG. 4. Dissociation of $\mathrm{O}_{3}$ into diatomic oxygen, $\mathrm{O}_{2}$. Horizontal error bars estimate the maximum experimental inaccuracy for assigning a data point to a specific instant of time. Vertical error bars and red shading refer to $95 \%$ confidence interval of data and fit, respectively. Red line indicates fitted temporal evolution using a fit function as explained in the main text. (a) Right: The spectrogram, $S(E, t)$, consisting of all measured spectra exhibiting the temporal evolution of intramolecular dynamics of ozone is shown as a function of XUV-DUV time delay (negative delays: XUV precedes DUV), using a logarithmic color scale. Left: Typical $\mathrm{O}_{3}$ photoelectron spectrum and the energy domains relevant for data evaluation. These correlate with state-specific cation states (see main text). At zero delay the buildup of $B$ state-associated photoelectrons (uppermost box: 90-93 eV) is accompanied by a decrease of $X$ state-related photoelectrons (second and fourth boxes: 79.5-81 eV and 86.5-88 eV). After $\sim 20$ fs a rise of photoelectrons emanating from diatomic oxygen, $\mathrm{O}_{2}$, shows up (third box: 82.5-85 $\mathrm{eV}$ ), proving the successful dissociation of ozone. (b) Energy-integrated number of ground-state-related photoelectrons: Depletion of ground state is accomplished between -10 and $10 \mathrm{fs}$ with a temporary increase around $\sim 25 \mathrm{fs}$ most likely coming from $\mathrm{O}_{2}$-related photoelectron contamination. (c) Energy-integrated number of $\mathrm{O}_{2}$-related photoelectrons: Successful photolysis from $\mathrm{O}_{3}$ into $\mathrm{O}_{2}$ is accomplished for the first time between 10 and $20 \mathrm{fs}$, and a second time between 30 and $40 \mathrm{fs}$ (first and second kink). For explanation see main text. 

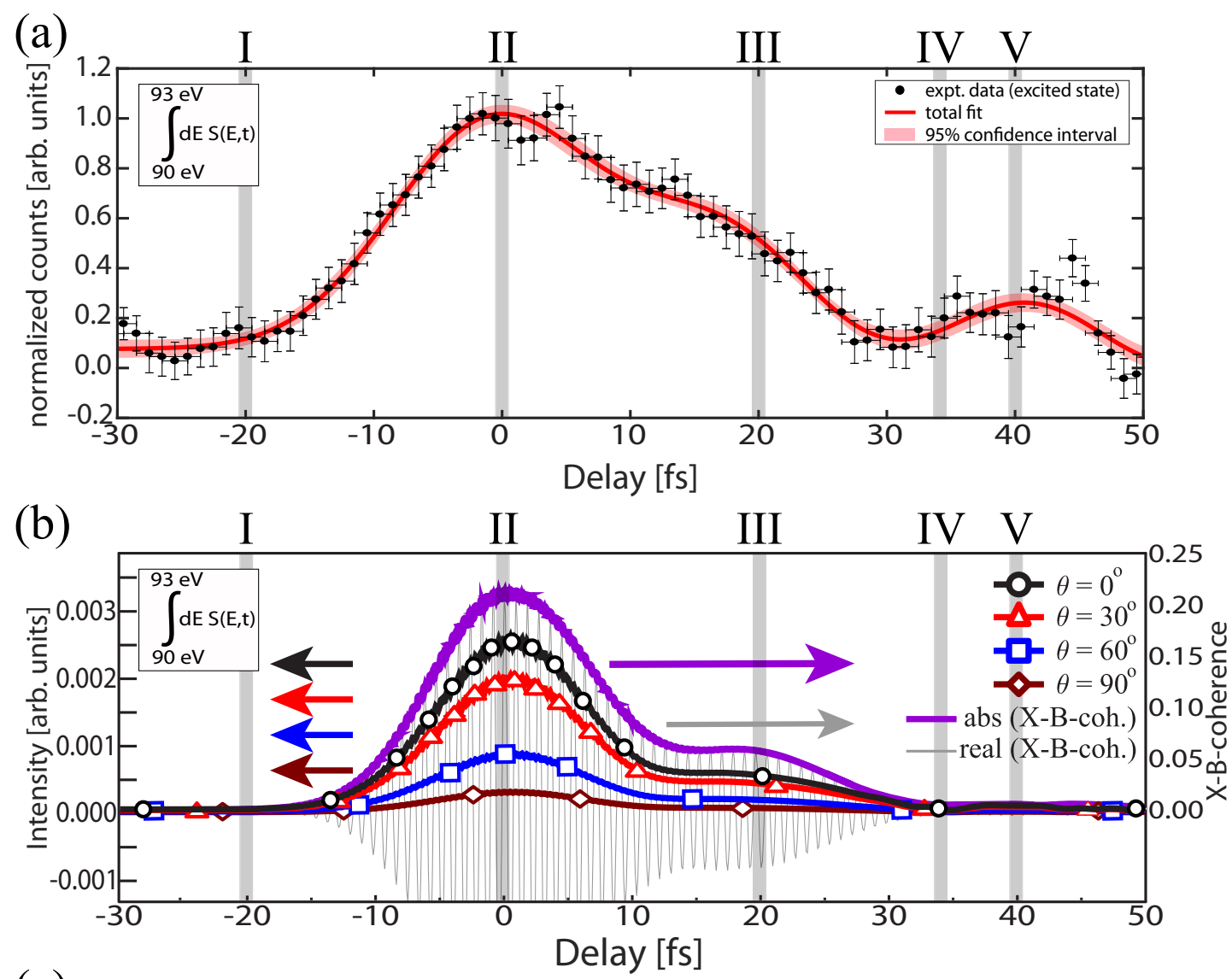

(c)
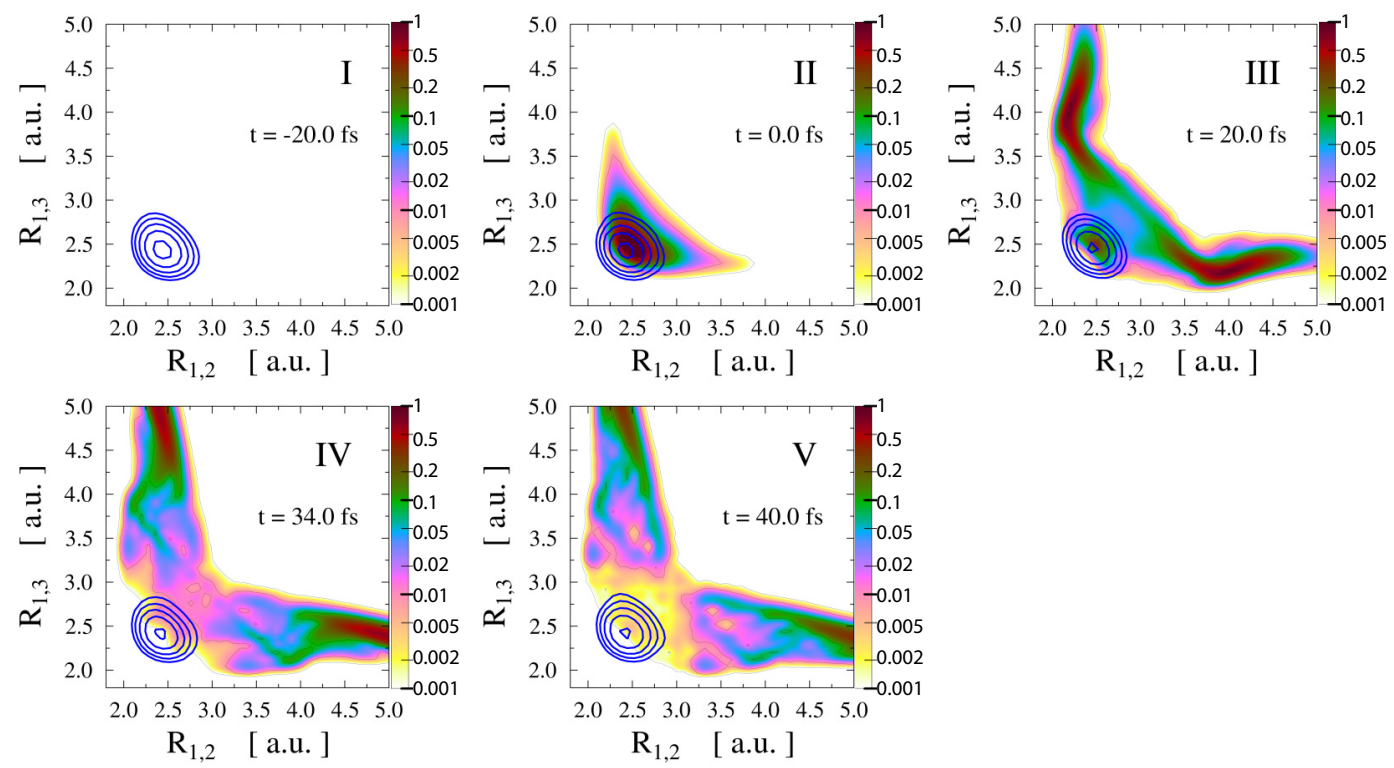

FIG. 5. Temporal evolution of $B$-state-related photoelectron emissions and intramolecular dynamics obtained from MCTDH modeling. (a) Measured temporal evolution of $B$-state-related photoelectrons integrated over the energy domain relevant for cation states emerging from the excited $B$ state (90-93 eV) (for details regarding the dynamics see main text). (b) Simulated temporal evolution of $B$-state-associated photoelectrons (brown, blue, red, and black curves) integrated over the same energy range as in (a) and contrasted with the temporal evolution of the absolute value (purple curve) and the real part (light gray curve) of the electronic coherence between the ground $X$ state and the excited $B$ state. (c) Satisfactory qualitative agreement between (a) and (b) unveils the electronic and nuclear dynamics in ozone via comparison to MCTDH NWP propagation for five exemplary instants of time (I-V). Way before temporal overlap ( -20 fs) there is no population on the $B$-state PES (I). At 0 fs, maximum excitation is achieved and located in close vicinity of the FC point (II). Around $20 \mathrm{fs}$, parts of the NWP have already dissociated, while another part of the NWP returns to the FC point for the first time (III). Minimum NWP density at the FC point is obtained around $34 \mathrm{fs}$ (IV), before a second revival of NWP density is observed around $40 \mathrm{fs}$ (V), being accompanied by repeated dissociation. 
excited state of $\tau_{d}=(10.0 \pm 5.4)$ fs. On top of the Gaussianconvolved decay function we observe a residual oscillation in the number of photoelectrons as a function of time delay. The oscillation period is determined to $\tau_{\mathrm{osc}}=(22.6 \pm 1.7)$ fs. To contrast our observations with full quantum dynamics simulations based on the Born-Oppenheimer approximation, we solved the nonrelativistic Schrödinger equation for a 10fs DUV pulse using the multiconfigurational time-dependent Hartree (MCTDH) approach [25-27]. Starting from these calculations we simulate the time-resolved photoelectron angular distribution for an isotropically oriented molecular ozone ensemble based on Dyson orbitals calculated with the MOLPRO package [28] and exploit electronic wave functions only in the near vicinity of the FC point. For more computational details, see Refs. [8,18,29].

Figure 5(b) depicts the energy-integrated outcome (90$93 \mathrm{eV}$ ) of our simulation calculated for several detection directions indicated by the angle $\theta$ between the XUV polarization and the electron time-of-flight detector (TOF) axis, clearly predicting a high asymmetry factor. Along with these, Fig. 5(b) also depicts the temporal evolution of the electronic coherence between the $X$ and $B$ states (purple and light gray lines) that is induced by the DUV pump pulse as stated above and closely resembles the temporal evolution of the $B$-staterelated photoelectron yield [Figs. 5(a) and 5(b)]. Comparison between Figs. 5(a) and 5(b) yields qualitative agreement, especially for the relative positions of the most interesting features [indicated by the five vertical bars at $-20 \mathrm{fs}$ (I), 0 fs (II), 20 fs (III), 34 fs (IV), and 40 fs (V)]. Due to the good accordance between theory and experiment, we can now relate these features to snapshots of the excited wave packet density for five different times as obtained from our MCTDH simulation [labeled I-V in Fig. 5(c)]. Figure 5(c) shows the $\alpha$ degree of freedom integrated NWP density on the $B$-state PES as a function of the bond lengths between the central oxygen atom and either one of the terminal $\mathrm{O}$ atoms, $R_{1,2}$ and $R_{1,3}$, respectively (see Sec. 9 of the Supplemental Material [9] for more snapshots). The NWP was calculated explicitly considering the three-dimensional nature of the PES and integration over the $\alpha$ degree of freedom was conducted subsequently. Starting at times before the DUV interacts with the ozone molecule ( $-20 \mathrm{fs}$ ) gives an unpopulated $B$-state PES [subplot I in Fig. 5(c)]. As soon as the DUV pulse interacts with the molecule ( $0 \mathrm{fs}$ ), the $B$ state PES is partly populated due to resonant excitation and most of the NWP resides close to the FC point [subplot II in Fig. 5(c)]. From this moment on its spatial and temporal evolution is dictated by the $B$ state PES and leads to fission of the NWP as it propagates towards a valley-ridge inflection point of the $B$-state PES, where it segregates into three parts. Two of them start dissociating while the remaining component returns to the FC point at around 20 fs [subplot III in Fig. 5(c)]. Once it has returned, the process repeats and the NWP revisits the valley-ridge inflection point in such a way that the NWP density around the FC point is almost zero at $\sim 34$ fs [subplot IV in Fig. 5(c)]. Since the splitting of the NWP is repeated a second time, part of it returns to the FC point again, where its density has built itself up once more at 40 fs [subplot $\mathrm{V}$ in Fig. 5(c)]. The second recurrence of the NWP to the FC point is, of course, accompanied by another dissociating NWP, explaining the reduced amplitude of the second recurrence at $40 \mathrm{fs}$ and manifests itself as yet another increase in the number of $\mathrm{O}_{2}$ related photoelectrons at the delay time $\tau=40$ fs [Fig. 4(c)].

In the reported configuration, the NWP density around the FC point can directly be probed by time-resolved photoelectron spectroscopy experiments owing to the strong dipole transition coupling between the PES of the neutral ozone, $\mathrm{O}_{3}$, and the PES of its singly ionized cation, $\mathrm{O}_{3}{ }^{+}$, at the $\mathrm{FC}$ point during XUV photoionization, as well as to its smaller coupling for configurations far away from the FC point. The observed time dependence of the $B$-state photoelectron emission is a direct consequence of the evolving wave packet on the $B$ state visiting different areas of its PES, which in turn periodically modulates the spatial overlap between the wave function of the neutral $\mathrm{O}_{3}$ and that of any state of the cation and thus the XUV induced transition rate.

\section{CONCLUSION AND OUTLOOK}

In conclusion, we report the observation of the convolved femtosecond time-scale electronic and nuclear dynamics of ozone, triggered by $\sim 10-\mathrm{fs}, \sim 2-\mu \mathrm{J}$ DUV pulses, via an extremely demanding UV-pump-XUV-probe experiment. We find experimental evidence for molecular fragmentation being accompanied by $\sim 22$-fs periodic oscillations of parts of the excited $B$-state-related nuclear wave packet around the $\mathrm{FC}$ point, a striking signature of ultrafast coherent photochemistry. Satisfactory agreement between the experimental data and numerical calculations based on the MCTDH approach allows us to derive a microscopic interpretation that links these oscillations to closed trajectories of the NWP on the $B$-state PES. Despite many approximations, our simulations capture the dominant effects of the experimental findings. Among these approximations, the negligence of contributions from NWPs far away from the FC point as well as the hypothesis of an isotropic ozone sample should be mentioned. Nonetheless, based on the already satisfactory qualitative accordance of theory and experiment we anticipate that inclusion of these aspects in future theoretical treatments will lead to only subtle corrections to the overall molecular dynamics with the prospect of quantitative predictive power, if proper molecular alignment, refined photoionization transition probabilities, and the full vibrational motion can be included. As pointed out above, a spatially narrow photoionization dipole transition coupling allows for the direct study of the excited NWP density close to the FC point.

Moreover, regarding the complexity of the PES of ozone exhibiting a variety of conical intersections (CIs) [30], it was shown that nonadiabatic crossings do happen quite frequently [3,31-33], but are less important in first-order approximation and on ultrashort (few-fs) time scales, rendering their inclusion unnecessary for an already realistic description of the NWP kinetics. This especially holds true for the photodissociation channel, which is found to be determined solely by the topography of the $B$-state PES although it possesses CIs with diabatic $R$ and $A$ states [31]. The presence of the CI with the $R$ state is at the origin of the highly structured Huggins band, which is the lowest part in energy of the Hartley band. But it is well known that the coupling between the $B$ and $R$ states is weak: the predissociation lifetime of the metastable 
states corresponding to the Huggins band has been estimated to be in the range of $1-4$ ps [34]. The $R$ state has initially been included in the simulations as well, but turned out to have a negligible impact on the few-femtosecond time-scale dynamics of ozone photodissociation.

The development of even shorter, more intense and tuneable DUV sources [21,22,35] should open up the possibility to control the ratio between the dissociating and vibrational parts of the NWP and might even allow us to monitor and control nonadiabatic crossings between the $B$ state and its intersecting $A$ and $R$ states. To derive their signature in time-resolved photoelectron spectroscopy experiments these diabatic states should additionally be considered in future quantum dynamical calculations. Based upon the proposed scheme of Kowalewski and co-workers [36,37], for example, these studies would not be limited to the ozone molecule, but could also be extended to biologically relevant molecules like peptides, proteins, or DNA, which absorb in the very same spectral range as ozone does. First steps towards this goal have been taken and temporal shaping of laser pulses in the DUV spectral domain promises to give direct control over the NWP's motion as was shown theoretically by Keefer et al. using the example of the RNA nucleobase uracil [38]. The systematic use of quantum coherence to enhance the rates of chemical reactions is one important challenge in modern chemistry [39-51]. The possibility to create not only nuclear, but also electronic wave packets in molecules, as in the present work, will allow one to play with both the nuclear and electronic interferences and thus to work at the most elementary level of chemical processes. The creation of a neutral electronic wave packet is also an advantage, since it means that the pump pulse did not alter the initial molecules.

\section{ACKNOWLEDGMENTS}

We acknowledge generous infrastructural support from F. Krausz. This work was supported by the Max Planck Society, the Deutsche Forschungsgemeinschaft Cluster of Excellence: Munich Centre for Advanced Photonics (http: //www.munich-photonics.de), the Austrian Science Foundation project NEXTLITE (F049 and P23359-N16) and LASERLAB-EUROPE (Grant Agreement No. 654148, European Union's Horizon 2020 research and innovation program). R.K. acknowledges a Consolidator Grant from the European Research Council (ERC-2014-CoG AEDMOS). This research was supported by EU-funded Hungarian Grant No. EFOP-3.6.2-16-2017-00005.

Experimental studies and analysis of experimental and theoretical signatures were carried out by T.L., V.S., M.O., J.R., B.B., and W.H., and supervised by R.K. Theory and modeling were performed by Á.V., G.J.H., and P.D., supported by F.G., B.L., and D.L. Customized XUV optics were provided by A.G. Customized NIR attenuating-DUV reflecting optics were provided by O.R. The ozone distillation setup was developed and refined by M.F., S.H., A.S., M.J., T.L., C.J., and M.S. The manuscript was written by T.L. All authors discussed the results and commented on the paper. The authors declare no competing financial interests.
[1] Z.-W. Qu, H. Zhu, S. Y. Grebenshchikov, and R. Schinke, The triplet channel in the photodissociation of ozone in the Hartley band: Classical trajectory surface hopping analysis, J. Chem. Phys. 122, 191102 (2005).

[2] Z.-W. Qu, H. Zhu, S. Y. Grebenshchikov, and R. Schinke, The photodissociation of ozone in the Hartley band: A theoretical analysis, J. Chem. Phys. 123, 074305 (2005).

[3] Z.-W. Qu, H. Zhu, M. Tashiro, and R. Schinke, The Huggins band of ozone: Unambiguous electronic and vibrational assignment, J. Chem. Phys. 120, 6811 (2004).

[4] Z.-W. Qu, H. Zhu, and R. Schinke, The ultra-violet photodissociation of ozone revisited, Chem. Phys. Lett. 377, 359 (2003).

[5] R. Schinke and G. C. McBane, Photodissociation of ozone in the Hartley band: Potential energy surfaces, nonadiabatic couplings, and singlet/triplet branching ratio, J. Chem. Phys. 132, 044305 (2010).

[6] E. Baloïtcha and G. G. Balint-Kurti, Theory of the photodissociation of ozone in the Hartley continuum: Potential energy surfaces, conical intersections, and photodissociation dynamics, J. Chem. Phys. 123, 014306 (2005).

[7] R. Hossaini et al., The increasing threat to stratospheric ozone from dichloromethane, Nat. Commun. 8, 15962 (2017).

[8] G. J. Halász, A. Perveaux, B. Lasorne, M. A. Robb, F. Gatti, and A. Vibok, Coherence revival during the attosecond electronic and nuclear quantum photodynamics of the ozone molecule, Phys. Rev. A 88, 023425 (2013).
[9] See Supplemental Material at http://link.aps.org/supplemental/ 10.1103/PhysRevA.99.063405 for brief description of the material.

[10] C. Arnold, O. Vendrell, and R. Santra, Electronic decoherence following photoionization, Phys. Rev. A 95, 033425 (2017).

[11] M. Vacher, M. J. Bearpark, M. A. Robb, and J. P. Malhado, Electron Dynamics upon Ionization of Polyatomic Molecules: Coupling to Quantum Nuclear Motion and Decoherence, Phys. Rev. Lett. 118, 083001 (2017).

[12] K. Siegbahn, Electron spectroscopy - an outlook, J. Electron Spectrosc. Relat. Phenom. 5, 3 (1974).

[13] M. Nisoli et al., Attosecond electron dynamics in molecules, Chem. Rev. 117, 10760 (2017).

[14] O. Travnikova et al., Subfemtosecond Control of Molecular Fragmentation by Hard X-Ray Photons, Phys. Rev. Lett. 118, 213001 (2017).

[15] E. W. Schlag et al., Distal charge transport in peptides, Angew. Chem. Int. Ed. Engl. 46, 3196 (2007).

[16] D. V. Vyalikh et al., Charge Transport in Proteins Probed by Resonant Photoemission, Phys. Rev. Lett. 102, 098101 (2009).

[17] C. Wan et al., Femtosecond dynamics of DNA-mediated electron transfer, Proc. Natl. Acad. Sci. USA 96, 6014 (1999).

[18] P. Decleva et al., Attosecond electronic and nuclear quantum photodynamics of ozone monitored with time and angle resolved photoelectron spectra, Sci. Rep. 6, 36613 (2016). 
[19] R. B. Varillas et al., Microjoule-level, tunable sub-10 fs UV pulses by broadband sum-frequency generation, Opt. Lett. 39, 3849 (2014).

[20] Y. Kida and T. Kobayashi, Generation of sub-10 fs ultraviolet Gaussian pulses, J. Opt. Soc. Am. B 28, 139 (2011).

[21] T. Kanai et al., Generation of subterawatt sub-10-fs blue pulses at $1-5 \mathrm{kHz}$ by broadband frequency doubling, Opt. Lett. 28, 1484 (2003).

[22] P. Baum, S. Lochbrunner, and E. Riedle, Tunable sub-10-fs ultraviolet pulses generated by achromatic frequency doubling, Opt. Lett. 29, 1686 (2004).

[23] R. F. Reilman, A. Msezane, and S. T. Manson, Relative intensities in photoelectron spectroscopy of atoms and molecules, J. Electron Spectrosc. Relat. Phenom. 8, 389 (1976).

[24] C. M. Oana and A. I. Krylov, Dyson orbitals for ionization from the ground and electronically excited states within equationof-motion coupled-cluster formalism: Theory, implementation, and examples, J. Chem. Phys. 127, 234106 (2007).

[25] H.-D. Meyer, U. Manthe, and L. S. Cederbaum, The multiconfigurational time-dependent Hartree approach, Chem. Phys. Lett. 165, 73 (1990).

[26] M. H. Beck, A. Jäckle, G. A. Worth, and H.-D. Meyer, The multiconfiguration time-dependent Hartree (MCTDH) method: A highly efficient algorithm for propagating wavepackets, Phys. Rep. 324, 1 (2000).

[27] G. A. Worth, M. H. Beck, A. Jäckle, and H.-D. Meyer, The MCTDH Package, Version 8.2, (2000). Meyer H.-D., Version 8.3 (2002), Version 8.4 (2007). Current version: 8.4 .12 (2016). See http://mctdh.uni-hd.de/.

[28] H.-J. Werner et al., Molpro, WIREs Comput Mol Sci 2, 242 (2012).

[29] A. Perveaux et al., Monitoring the birth of an electronic wavepacket in a molecule with attosecond time-resolved photoelectron spectroscopy, J. Phys. Chem. A 118, 8773 (2014).

[30] Conical Intersections. Theory, Computation and Experiment, edited by W. Domcke, D. R. Yarkony, and H. Köppel (World Scientific, Singapore, 2011), Vol. 17.

[31] S. Y. Grebenshchikov, Z.-W. Qu, H. Zhu, and R. Schinke, New theoretical investigations of the photodissociation of ozone in the Hartley, Huggins, Chappuis, and Wulf bands, Phys. Chem. Chem. Phys. 9, 2044 (2007).

[32] Z.-W. Qu et al., The Huggins band of ozone: A theoretical analysis, J. Chem. Phys. 121, 11731 (2004).

[33] Z.-W. Qu, H. Zhu, and R. Schinke, Infrared spectrum of cyclic ozone: A theoretical investigation, J. Chem. Phys. 123, 204324 (2005).

[34] S. A. Ndengué et al., Comparison of the Huggins band for six ozone isotopologues, J. Phys. Chem. A 116, 12260 (2012).
[35] P. Baum, S. Lochbrunner, and E. Riedle, Generation of tunable 7-fs ultraviolet pulses: Achromatic phase matching and chirp management, Appl. Phys. B 79, 1027 (2004).

[36] M. Kowalewski, K. Bennett, J. R. Rouxel, and S. Mukamel, Monitoring Nonadiabatic Electron-Nuclear Dynamics in Molecules by Attosecond Streaking of Photoelectrons, Phys. Rev. Lett. 117, 043201 (2016).

[37] M. Kowalewski et al., Simulating coherent multidimensional spectroscopy of nonadiabatic molecular processes, Chem. Rev. 117, 12165 (2017).

[38] D. Keefer, S. Thallmair, S. Matsika, and R. de Vivie-Riedle, Controlling photorelaxation in uracil with shaped laser pulses: A theoretical assessment, J. Am. Chem. Soc. 139, 5061 (2017).

[39] F. Gatti, Molecular dynamics simulated by photons, Nature (London) 557, 641 (2018).

[40] W. Schweinberger et al., Waveform-controlled near-singlecycle milli-joule laser pulses generate sub-10 nm extreme ultraviolet continua, Opt. Lett. 37, 3573 (2012).

[41] P. B. Corkum, C. Rolland, and T. Srinivasan-Rao, Supercontinuum Generation in Gases, Phys. Rev. Lett. 57, 2268 (1986).

[42] S. P. Le Blanc, S. C. Rae, K. Burnett, and R. Sauerbrey, Spectral blue shifting of a femtosecond laser pulse propagating through a high-pressure gas, J. Opt. Soc. Am. B 10, 1801 (1993).

[43] M. Kolesik, J. V. Moloney, and M. Mlejnek, Unidirectional Optical Pulse Propagation Equation, Phys. Rev. Lett. 89, 283902 (2002).

[44] J. E. Midwinter and J. Warner, The effects of phase matching method and of uniaxial crystal symmetry on the polar distribution of second-order non-linear optical polarization, Br. J. Appl. Phys. 16, 1135 (1965).

[45] R. E. Newnham, Properties of Materials: Anisotropy, Symmetry, Structure (Oxford Univiversity Press, Oxford, 2006).

[46] D. A. Kleinman, Nonlinear dielectric polarization in optical media, Phys. Rev. 126, 1977 (1962).

[47] R. W. Boyd, Nonlinear Optics (Elsevier, Amsterdam, 2008).

[48] R. Kienberger et al., Steering attosecond electron wave packets with light, Science (New York, N.Y.) 297, 1144 (2002).

[49] O. Razskazovskaya et al., Carrier frequency tuning of few-cycle light pulses by a broadband attenuating mirror, Appl. Opt. 56, 8978 (2017).

[50] R. Trebino et al., Measuring ultrashort laser pulses in the timefrequency domain using frequency-resolved optical gating, Rev. Sci. Instrum. 68, 3277 (1997).

[51] M. J. Jobst, Ph.D. thesis, Technische Universität München, 2014. 